\title{
Cell-free DNA in medium is associated with the maturation ability of in vitro cultured oocytes
}

\author{
Yasuhisa MUNAKATA ${ }^{1}$, Koumei SHIRASUNA $^{1)}$, Takehito KUWAYAMA ${ }^{1)}$ and Hisataka IWATA ${ }^{1)}$ \\ ${ }^{1)}$ Department of Animal Reproduction, Tokyo University of Agriculture, Kanagawa 243-0034, Japan
}

\begin{abstract}
Follicular fluid contains cell-free DNA (cfDNA), which may serve as a useful biomarker of oocyte ability. The present study evaluates whether nuclear and mitochondrial cfDNAs in conditioned oocyte growth medium determine the quality of oocytes cultured in vitro. Oocyte and granulosa cell complexes (OGCs) derived from early antral follicles of gilt ovaries were cultured for 14 days and the amount of cfDNA and lactate concentration in the conditioned culture medium were measured and compared to evaluate oocyte maturation ability. The amount of nuclear cfDNA, but not mitochondrial cfDNA, strongly correlated with the number of dead cells in OGCs. Furthermore, low mitochondrial cfDNA content and high lactate concentration in the medium was associated with high maturation ability of oocytes cultured in vitro. In conclusion, the amounts of nuclear and mitochondrial cfDNAs differentially reflect the conditions of OGCs, and low mitochondrial cfDNA, low glucose content, and high lactate concentration in the medium are associated with the proper maturation of oocytes.
\end{abstract}

Key words: In vitro cultured oocytes, Mitochondrial cfDNA, Nuclear cfDNA, Oocyte quality

(J. Reprod. Dev. 65: 171-175, 2019)

$\mathbf{I}_{\mathrm{c}}^{\mathrm{n}}$ n assisted reproductive technology, non-invasive markers of oocyte quality have been intensively investigated. Cell-free DNAs (cfDNAs) are extracellular DNA molecules that circulate in the blood and are associated with various pathological conditions of donors, including malignancy, heart disease, stroke, tissue-specific dysfunctions, and sepsis [1]. The high content of cfDNA in follicular fluid (FF) is associated with poor oocyte quality, high rate of fragmentation, and low rate of cleavage and pregnancy in the subsequent embryonic development process [2, 3]. Furthermore, the supplementation of culture medium with cfDNA $(4 \mathrm{mg} / \mathrm{ml})$ was shown to induce apoptosis in granulosa cells (GCs) [1]. However, the levels of cfDNA added to the medium was in the order of milligrams, which is significantly higher than that present in FF $(2.2-16.4 \mu \mathrm{g} /$ ml) [4]. In addition, cfDNA in FF is derived from circulation and/ or follicular cells. Therefore, it is possible that the patients with severe physiological conditions have both low-quality oocytes and high content of cfDNA in circulation and FFs. Although cfDNA is believed to be derived from apoptotic and dead cells, it has been reported that breast cancer cell lines actively secrete cfDNA [5]. Furthermore, live GCs secrete mitochondrial cfDNA in response to mitochondrial uncoupler treatment [4]. In this context, the in vitro growth (IVG) system of oocytes is useful to evaluate the relationship between the amount of cfDNA derived from native follicular cells and the quality of oocytes cultured in vitro. The recent development in the culture systems for oocytes of large animals have facilitated

Received: October 18, 2018

Accepted: January 12, 2019

Published online in J-STAGE: February 11, 2019

(C)2019 by the Society for Reproduction and Development

Correspondence: H Iwata (e-mail: hliwata@nodai.ac.jp)

This is an open-access article distributed under the terms of the Creative Commons Attribution Non-Commercial No Derivatives (by-nc-nd) License. (CC-BY-NC-ND 4.0: https://creativecommons.org/licenses/by-nc-nd/4.0/) the in vitro cultivation of oocytes derived from early antral follicles (EAFs) in manner similar to the in vivo cultivation of the oocyte collected from large antral follicles [6]. In the present study, we cultured oocytes and GC complexes (OGCs) derived from EAFs for two weeks and examined the nuclear and mitochondrial cfDNA content in the conditioned medium, the number and survival rate of GCs surrounding the oocytes, and the maturation ability of the oocytes cultured in vitro.

\section{Materials and Methods}

\section{Collection of OGCs from EAFs}

Ovaries were collected from prepubertal gilts slaughtered at a local abattoir for public consumption and transported to the laboratory at approximately $37^{\circ} \mathrm{C}$ in phosphate-buffered saline (PBS) containing antibiotics $(100 \mu \mathrm{g} / \mathrm{ml}$ streptomycin and $10 \mathrm{IU} / \mathrm{ml}$ penicillin) within a period of $1 \mathrm{~h}$. The study was approved by the ethics committee for animal experiments of the Tokyo University of Agriculture (Tokyo, Japan). Ovarian cortical tissues were excised from the ovarian surface under a stereomicroscope, and OGCs were collected from EAFs (0.5-0.7 $\mathrm{mm}$ in diameter). OGCs containing oocytes with diameters in the range of $90-100 \mu \mathrm{m}$ were selected for the experiments.

\section{Chemicals and media}

All reagents were purchased from Nacalai Tesque (Kyoto, Japan), unless otherwise stated. OGCs were cultured in vitro (IVG medium) in $\alpha$-minimum essential medium ( $\alpha$-MEM; Sigma-Aldrich, St. Louis, MO, USA) supplemented with $10 \mathrm{mM}$ taurine, $1 \mu \mathrm{g} / \mathrm{ml}$ of 17ß-estradiol, $0.1 \mathrm{mAU} / \mathrm{ml}$ of follicle-stimulating hormone (Kawasaki Mitaka, Tokyo, Japan), 2 mM hypoxanthine (Sigma-Aldrich), 1\% insulin-transferrin-selenium (Gibco BRL, Grand Island, NY, USA), $3 \mathrm{mg} / \mathrm{ml}$ bovine serum albumin (Fraction V), and antibiotics. The medium used for oocyte nuclear maturation (in vitro maturation, IVM 
medium) was prepared based on Medium 199 (Gibco) supplemented with $0.5 \mathrm{mM}$ l-cysteine, $0.9 \mathrm{mM}$ sodium pyruvate, $1 \mathrm{mM}$ l-glutamine, $10 \mathrm{ng} / \mathrm{ml}$ of epidermal growth factor, $5 \%$ fetal calf serum, porcine $\mathrm{FF}(\mathrm{pFF}, 10 \% \mathrm{v} / \mathrm{v}), 10 \mathrm{IU} / \mathrm{ml}$ of equine chorionic gonadotropin (ASKA Pharma, Tokyo, Japan), and $10 \mathrm{IU} / \mathrm{ml}$ of human chorionic gonadotropin (Fuji Pharma, Tokyo, Japan).

\section{Preparation of gel culture system for IVG}

Polyacrylamide gels (PAGs) were prepared as described in a previous report [7]. In brief, PAG was prepared following the general method for western blotting. The stiffness of PAG was adjusted with a combination of $10 \%(\mathrm{v} / \mathrm{v})$ acrylamide solution and $0.3 \%(\mathrm{v} / \mathrm{v})$ of $\mathrm{N}, \mathrm{N}$ '-methylenebisacrylamide solution. The gel sheets were washed and incubated in PBS overnight. PAGs were hollowed out from the gel sheet, placed at the bottom of 96-well plates, and incubated in IVG medium overnight for equilibration. Before the experiments were carried out, the medium was replaced with fresh IVG medium. The thickness of the PAG was $1 \mathrm{~mm}$.

\section{In vitro culture of $O G C s$}

OGCs were individually cultured in $200 \mu 1$ of culture medium in 96-well plates (Falcon 353072; BD Biosciences, Franklin Lakes, NJ, USA) for 14 days. The wells of 96-well plates were paved with polyacrylamide gel. Every two days, the number of OGCs forming an antrum was counted, and half of the medium was replaced with fresh medium on day 4,8 , and 12 . At the end of the culture period (14 days), OGCs with an antrum cavity were analyzed. IVG was performed at $38.5^{\circ} \mathrm{C}$ in $5 \% \mathrm{CO}_{2}$ and $95 \%$ air.

\section{GC count and survival rate of OGCs cultured in vitro}

After IVG, oocytes with GCs were separated from the remaining oocytes using a fine-pulled Pasteur pipette, and the GC mass was separated in a cell dispersion cocktail (Accumax; Innovative Cell Technologies, San Diego, CA, USA). The total cell number was calculated based on the volume and concentration of cellular suspension using a hemocytometer. The survival rate of GCs was determined using trypan blue staining for observation under a microscope (Olympus, Tokyo, Japan).

\section{IVM of oocytes}

At the end of IVG period (14 days), oocytes and the small number of surrounding cumulus cells were individually cultured for $44 \mathrm{~h}$. The oocytes were eventually denuded from the surrounding cells, fixed with $4 \%$ paraformaldehyde, and mounted on glass slides in an anti-fade reagent containing 4',6-diamidino-2-phenylindole (DAPI; ProLong gold antifade reagent with DAPI; Invitrogen, Carlsbad, CA, USA), followed by observation under an inverted fluorescence microscope (Olympus). The oocytes that reached metaphase II were defined as competent oocytes and those that failed to reach metaphase II were defined as non-competent oocytes. IVM was performed at $38.5^{\circ} \mathrm{C}$ in $5 \% \mathrm{CO}_{2}$ and $95 \%$ air.

\footnotetext{
Measurement of mitochondrial and nuclear cfDNA content in conditioned culture medium

Before assessment, the culture medium was transferred to a microtube and centrifuged $(5,000 \times \mathrm{g}$ for $5 \mathrm{~min})$ to remove cel-
}

lular debris. The supernatant was mixed with an equal volume of DNA extraction buffer (final concentration, $20 \mathrm{mM}$ Tris, $0.2 \%$ Tween-20, $0.2 \%$ Nonidet P-40) and incubated at $55^{\circ} \mathrm{C}$ for $30 \mathrm{~min}$ and $98^{\circ} \mathrm{C}$ for $10 \mathrm{~min}$. The mitochondrial and nuclear DNA contents were evaluated real-time polymerase chain reaction (PCR) using CFX Connect ${ }^{\mathrm{TM}}$ real-time PCR detection system (Bio-Rad, CA, USA) as previously described [8,9]. The primer set used for the mitochondrial genome was 5'-CGAGAGAGCACTTTCCAAGG-3' and 5'-CTAATTCGGGTGTTGGTGCT-3' (152 bp, NC_000845.1: $16613 \mathrm{bp}$;9,985-10,136), and that for nuclear DNA was 5'-AGCAGAATCAACACCATCGGT-3' and 5'-TGGCTCCACCCATAGAATGC-3' (154 bp, NC_010457.4: GCG glucagon).

\section{Measurement of mitochondrial DNA copy number in GCs}

After IVG, GCs were washed and the DNA in the cells was extracted with a DNA extraction buffer. Using the primer set targeting a single-copy gene (described above), the GC number in the sample was predicted, and the copy number of mitochondrial DNA was determined from the predicted GC number for each cell.

\section{Measurement of glucose and lactate concentration in conditioned culture medium}

After IVG, the culture medium was transferred into a microtube and centrifuged $(5,000 \times \mathrm{g}$ for $5 \mathrm{~min})$ to remove cellular debris. The supernatant was preserved at $-20^{\circ} \mathrm{C}$ until analysis. Glucose content in culture medium was analyzed with LabAssay ${ }^{\mathrm{TM}}$ Glucose (Wako Pure Chemical Industries, Osaka, Japan), while the lactate content was evaluated with a lactate assay kit (BioVision, Mountain View, CA, USA) following manufacturer's instructions.

\section{Statistical analysis}

Comparison between two categories was conducted with Student's $t$-test. Percentages were arcsine transformed before analysis. Correlations were calculated using SPSS (Version 17.0, Chicago, IL, USA). A value of $\mathrm{P}<0.05$ was considered statistically significant.

\section{Results}

Amount of nuclear cfDNA correlates with the survival rate of GCs in culture wells

The cfDNA is derived from dead or living cells $[5,10]$. Culture conditions are known to differentially affect the content of nuclear and mitochondrial cfDNAs in medium [4]. We, therefore, examined whether nuclear or mitochondrial cfDNA in the medium was derived from dead cells (Fig. 1, Arrow I). OGCs were cultured for 14 days and the relationship between cfDNA content in the spent medium and the number and survival rate of GCs in OGCs were examined. The data in scatter plots are shown in Fig. 2. We found a significant negative correlation between survival rate of GCs and nuclear cfDNA content (Fig. 2-D, $\mathrm{R}^{2}=0.279, \mathrm{P}<0.05$ ); however, no relationship was detected for other combinations (Figs. 2-A, 2-B, and 2-C and $\mathrm{R}^{2}=0.018,0.025$, and 0.125 , respectively). 


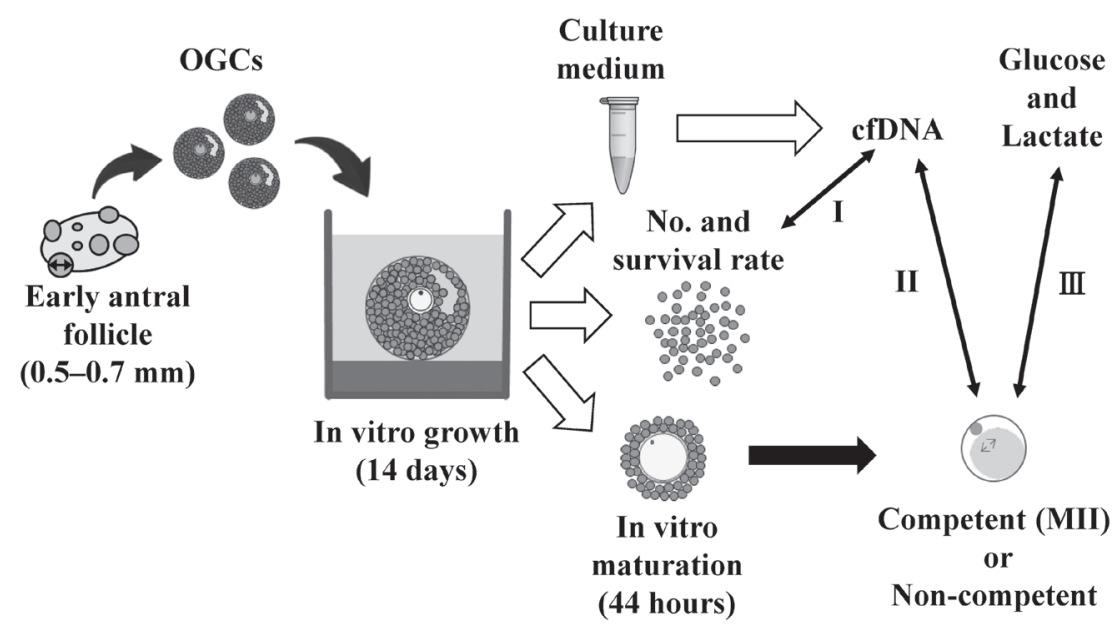

Fig. 1. Schematic design of oocytes and granulosa cell complexes (OGCs) cultured in vitro. OGCs collected from early antral follicles were individually cultured for 14 days. The number and survival rate of granulosa cells surrounding the oocytes grown in vitro were determined and the amount of nuclear and mitochondrial cfDNA in the conditioned culture medium was evaluated (Arrow I). The oocytes cultured in vitro were subjected to in vitro maturation for $44 \mathrm{~h}$, and the ability of nuclear maturation was compared by determining the amount of cfDNA (Arrow II) and glucose and lactate contents (Arrow $\amalg$ ) in the spent culture medium.
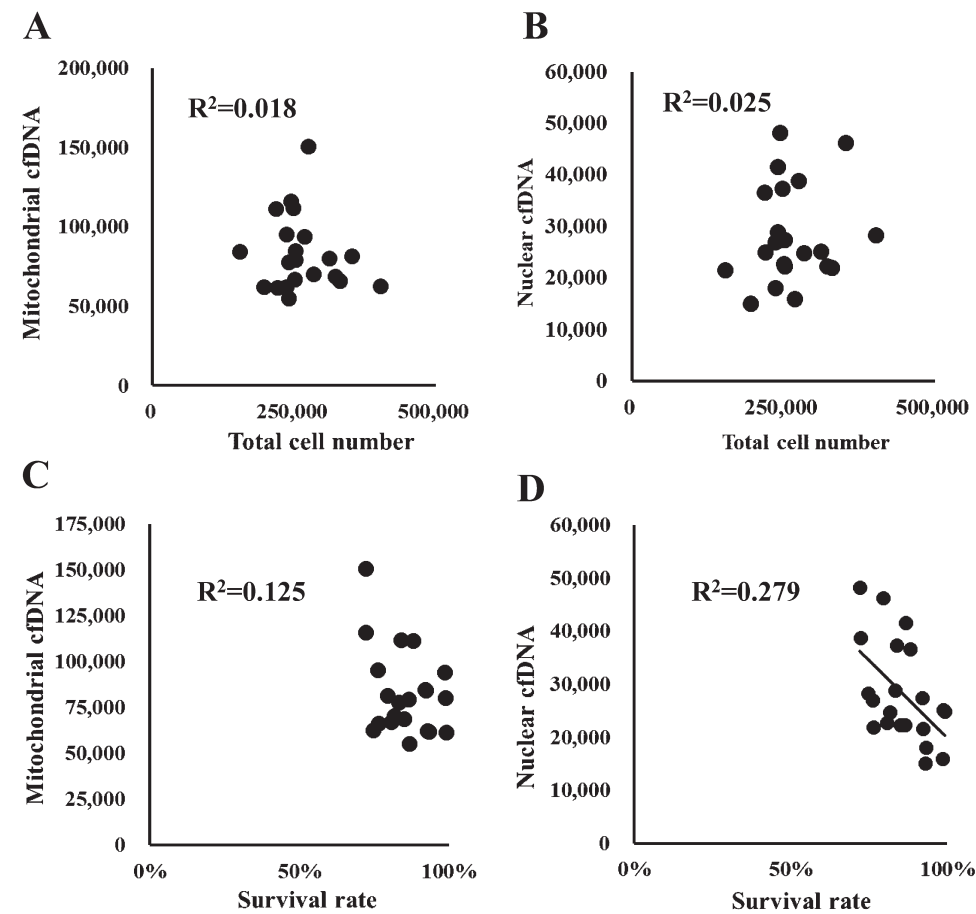

Fig. 2. Relationship between nuclear and mitochondrial cell-free DNA (cfDNA) and the number of total or dead granulosa cells in culture wells. Y-axis: copy number (in $200 \mu \mathrm{l}$ of medium) of either mitochondrial (A, C) or nuclear cfDNA (B, D); X-axis: number of total granulosa cells (A, B) or survival rate of granulosa cells $(\mathrm{C}, \mathrm{D})$. A significant correlation was detected between the dead cell number and amount of nuclear cfDNA in the medium $\left(\mathrm{D}, \mathrm{R}^{2}=0.279, \mathrm{P}<0.05\right)$.

High mitochondrial cfDNA content in conditioned culture medium correlates with the maturation ability of oocytes

We addressed the relationship between cfDNA content in the medium and the ability of oocytes to complete nuclear maturation in vitro. OGCs were cultured for 14 days, and the oocytes cultured in vitro were individually subjected to IVM to determine their ability to complete nuclear maturation (whether the oocyte could progress to metaphase II). In addition, the amount of nuclear and mitochondrial 
A

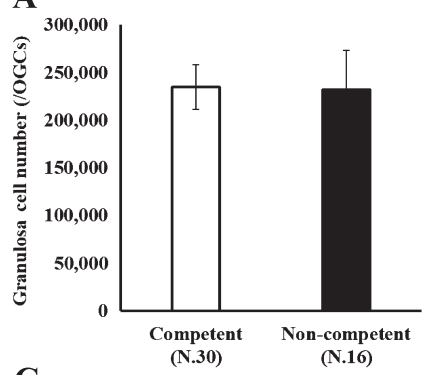

C

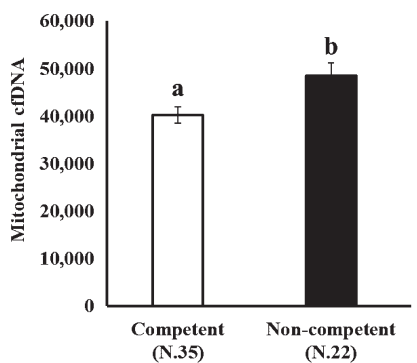

B

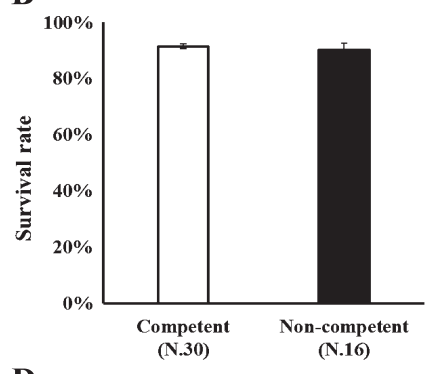

D

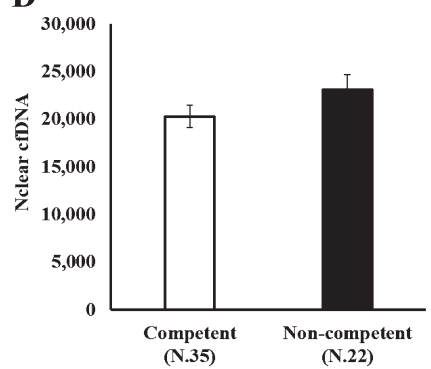

Fig. 3. Number and survival rate of granulosa cells of an oocyte and granulosa cell complexes (OGCs) (A, B) and the copy number of either mitochondrial or nuclear cfDNA in medium $(\mathrm{C}, \mathrm{D})$ was compared between competent and non-competent oocytes. OGCs collected from early antral follicles were individually cultured for 14 days. The oocytes grown in vitro were subjected to in vitro maturation for $44 \mathrm{~h}$ to determine their ability to undergo nuclear maturation. Copy number of mitochondrial and nuclear cfDNAs in the spent culture medium $(200 \mu \mathrm{l})$ was determined.

cfDNAs in the medium was compared between the competent and non-competent oocytes (Fig. 1, Arrow II). As shown in Fig. 3, no difference was observed in the number and survival rate of GCs between oocytes with different nuclear maturation abilities (Figs. 3-A, 3-B; competent and non-competent oocytes, oocytes that could reach metaphase II or not following IVM, respectively). The content of mitochondrial cfDNA was significantly higher in the medium containing non-competent oocytes than in the medium from competent oocytes (Fig. 3-C). We examined whether the difference in the mitochondrial cfDNA content in the medium was attributed to the variation in the number of mitochondria in GCs. The mitochondrial DNA copy number in GCs was $490.0 \pm 16.8$ (N.27) and $486.6 \pm$ 26.8 (N.15) for competent and non-competent oocytes, respectively, indicative of no significant difference $(\mathrm{P}=0.91)$.

Low glucose and high lactate concentration in culture medium are associated with high oocyte maturation ability

We addressed the relationship between glucose and lactate concentration in the medium and the ability of oocytes to complete nuclear maturation in vitro. OGCs were cultured for 14 days, and the oocytes cultured in vitro were individually subjected to IVM to determine their ability to undergo nuclear maturation. The concentrations of glucose and lactate in the medium were measured and compared between the competent and non-competent oocytes (Fig. 1, Arrow III). As shown in Fig. 4, high maturation ability was associated with low glucose and high lactate content in the medium.
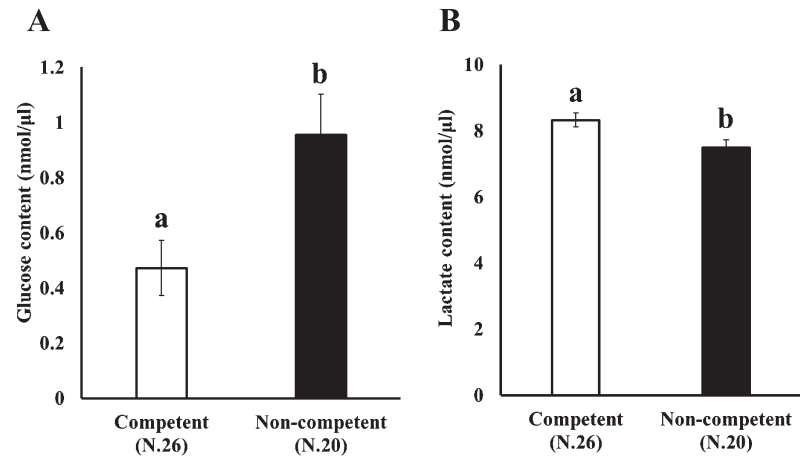

Fig. 4. Comparison of glucose and lactate contents in the medium between competent and non-competent oocytes. OGCs collected from early antral follicles were individually cultured for 14 days and glucose (A) and lactate (B) contents in the spent culture medium were examined. The oocytes grown in vitro were subjected to in vitro maturation for $44 \mathrm{~h}$ to determine their ability to undergo nuclear maturation.

\section{Discussion}

The present study demonstrates that nuclear cfDNA, but not mitochondrial cfDNA, in culture medium was derived from dead cells. The high competence of oocytes was associated with low mitochondrial cfDNA, low glucose, and high lactate content in the medium.

Here, we used culture conditions that allowed cultivation of oocytes with high developmental competence [7]. In a supplementary experiment, this culture method supported oocyte growth and allowed them to grow in vitro to reach a diameter of $117.6 \pm 0.8 \mu \mathrm{m}$ (N.24). The rate of blastulation following parthenogenetic activation was $15.5 \%$, consistent with that observed for the oocytes cultured in vivo.

The cfDNA comprises both nuclear and mitochondrial DNA, wherein the and mitochondrial cfDNA closely reflects the cfDNA from FFs [4]. It has been previously demonstrated that the induced mitochondrial dysfunction increased only mitochondrial cfDNA but not nuclear cfDNA secretion from GCs. Therefore, it is possible to speculate that differential factors affect nuclear and mitochondrial cfDNA secretion. The present study showed that the amount of nuclear cfDNA, but not mitochondrial cfDNA, closely correlated with dead cells (Fig. 2-D), suggesting that cellular apoptosis andnecrosis may be the key factors contributing to nuclear cfDNA in culture medium. The amount of cfDNA in FF may serve as a noninvasive marker of oocyte competence $[2,3,11]$. Here, we examine, for the fiest time, the relationship between the quality of oocytes cultured in vitro and the amount of "native" cfDNA in culture medium. Using in vitro culture conditions, we found that the high ability of nuclear maturation for oocytes cultured in vitro was associated with low mitochondrial cfDNA level in the medium (Fig. 3-C). In addition, the number and survival rates of GCs showed no difference between the two oocyte groups. These results suggest that the high level of mitochondrial cfDNA in the culture environment (native cfDNA of OGCs) reflects the adverse quality of oocytes. The molecular mechanisms underlying the relationship between high mitochondrial cfDNA secretion from 
OGCs and low nuclear maturation ability is still unclear. However, one possible reason for the high level of mitochondrial cfDNA may be the high frequency of GCs with mitochondrial dysfunction or differential mitochondrial hemostasis, as the mitochondrial content of GCs was similar in both oocyte groups. The glucose and lactate contents in medium are indicators of glycolytic activity. In the present study, high glucose consumption and lactate production in the medium were associated with the high competence of oocytes. Gene expression analysis of GCs revealed that the increase in the glycolytic activity during follicular development [12] and glycolysis in GCs plays an important role in follicle development [12-16]. Moreover, the relationship between glycolytic activity and nuclear maturation capacity was reported in cows $[17,18]$. Therefore, we hypothesize that the high glycolytic activity in GCs may cause high glucose consumption and lactate production and reduce the mitochondrial cfDNA content in culture medium, eventually contributing to the increase in the developmental ability of oocytes.

In conclusion, the amount of nuclear and mitochondrial cfDNA may differentially reflect the conditions of OGCs; low level of mitochondrial cfDNA, high glucose consumption of GCs, and high lactate content derived from follicular cells may serve as the markers of oocyte quality.

\section{Acknowledgments}

This study was supported by a Grant-in-Aid for Scientific Research C (KAKENHI, grant number: 16K07996) and a Grant-inAid for JSPS Fellows (KAKENHI, grant number: 18J12261) from the Japan Society for the Promotion of Science, and the Science Research Promotion Fund from the Promotion and Mutual Aid Corporation for Private Schools of Japan.

\section{References}

1. Boyapati RK, Tamborska A, Dorward DA, Ho GT. Advances in the understanding of mitochondrial DNA as a pathogenic factor in inflammatory diseases. F1000 Res 2017; 6: 169. [Medline] [CrossRef]

2. Traver S, Scalici E, Mullet T, Molinari N, Vincens C, Anahory T, Hamamah S. Cellfree DNA in human follicular microenvironment: new prognostic biomarker to predict in vitro fertilization outcomes. PLoS One 2015; 10: e0136172. [Medline] [CrossRef]
3. Guan Y, Zhang W, Wang X, Cai P, Jia Q, Zhao W. Cell-free DNA induced apoptosis of granulosa cells by oxidative stress. Clin Chim Acta 2017; 473: 213-217. [Medline] [CrossRef]

4. Kansaku K, Munakata Y, Itami N, Shirasuna K, Kuwayama T, Iwata H. Mitochondrial dysfunction in cumulus-oocyte complexes increases cell-free mitochondrial DNA. $J$ Reprod Dev 2018; 64: 261-266. [Medline] [CrossRef]

5. Wang W, Kong P, Ma G, Li L, Zhu J, Xia T, Xie H, Zhou W, Wang S. Characterization of the release and biological significance of cell-free DNA from breast cancer cell lines. Oncotarget 2017; 8: 43180-43191. [Medline]

6. Makita M, Ueda M, Miyano T. The fertilization ability and developmental competence of bovine oocytes grown in vitro. J Reprod Dev 2016; 62: 379-384. [Medline] [CrossRef]

7. Munakata Y, Kawahara-Miki R, Shirasuna K, Kuwayama T, Iwata H. Polyacrylamide gel as a culture substrate improves in vitro oocyte growth from porcine early antral follicles. Mol Reprod Dev 2017; 84: 44-54. [Medline] [CrossRef]

8. Sato D, Itami N, Tasaki H, Takeo S, Kuwayama T, Iwata H. Relationship between mitochondrial DNA copy number and SIRT1 expression in porcine oocytes. PLoS One 2014; 9: e94488. [Medline] [CrossRef]

9. Itami N, Shirasuna K, Kuwayama T, Iwata H. Resveratrol improves the quality of pig oocytes derived from early antral follicles through sirtuin 1 activation. Theriogenology 2015; 83: 1360-1367. [Medline] [CrossRef]

10. Bronkhorst AJ, Wentzel JF, Aucamp J, van Dyk E, du Plessis L, Pretorius PJ. Characterization of the cell-free DNA released by cultured cancer cells. Biochim Biophys Acta 2016; 1863: 157-165. [Medline] [CrossRef]

11. Da Broi MG, de Albuquerque FO, de Andrade AZ, Cardoso RL, Jordão Junior AA, Navarro PA. Increased concentration of 8-hydroxy-2'-deoxyguanosine in follicular fluid of infertile women with endometriosis. Cell Tissue Res 2016; 366: 231-242. [Medline] [CrossRef]

12. Munakata Y, Kawahara-Miki R, Shiratsuki S, Tasaki H, Itami N, Shirasuna K, Kuwayama T, Iwata H. Gene expression patterns in granulosa cells and oocytes at various stages of follicle development as well as in in vitro grown oocyte-and-granulosa cell complexes. J Reprod Dev 2016; 62: 359-366. [Medline] [CrossRef]

13. Harris SE, Adriaens I, Leese HJ, Gosden RG, Picton HM. Carbohydrate metabolism by murine ovarian follicles and oocytes grown in vitro. Reproduction 2007; 134: 415-424. [Medline] [CrossRef]

14. Collado-Fernandez E, Picton HM, Dumollard R. Metabolism throughout follicle and oocyte development in mammals. Int J Dev Biol 2012; 56: 799-808. [Medline] [CrossRef]

15. Makanji Y, Tagler D, Pahnke J, Shea LD, Woodruff TK. Hypoxia-mediated carbohydrate metabolism and transport promote early-stage murine follicle growth and survival. Am J Physiol Endocrinol Metab 2014; 306: E893-E903. [Medline] [CrossRef]

16. Anastácio A, Rodriguez-Wallberg KA, Chardonnet S, Pionneau C, Fédérici C, Almeida Santos T, Poirot C. Protein profile of mouse ovarian follicles grown in vitro. $\mathrm{Mol}$ Hum Reprod 2017; 23: 827-841. [Medline] [CrossRef]

17. Gutnisky C, Morado S, Dalvit GC, Thompson JG, Cetica PD. Glycolytic pathway activity: effect on IVM and oxidative metabolism of bovine oocytes. Reprod Fertil Dev 2013; 25: 1026-1035. [Medline] [CrossRef]

18. Sinha PB, Tesfaye D, Rings F, Hossien M, Hoelker M, Held E, Neuhoff C, Tholen E, Schellander K, Salilew-Wondim D. MicroRNA-130b is involved in bovine granulosa and cumulus cells function, oocyte maturation and blastocyst formation. J Ovarian Res 2017; 10: 37. [Medline] [CrossRef] 